\title{
IV. Der ätiologische Zusammenhang zwischen Entzündung des Zahnfleisches und anderweitigen Krankheiten.
}

Autorreferat nach einem im polnischen Aerzte-Verein am 23. Juni 1881 gehaltenen Vortrag, Przeglad lekarski Krakowski No. 17, 1881.

$$
\text { Von }
$$

Dr. v. Kaczorowski-Posen.

\author{
(Fortsetzung aus No. 33.)
}

Es soll im Nachstehenden nur der grobe Versuch gemacht werden, ex juvantibus eines einfachen Praktikers die Wege zu erschliessen, auf dem sich im Zahnfleisch gehegte Krankheitserreger auf andere Organe in störender Weise übertragen können. Der nächste Weg ihrer Weiterverbreitung wäre natürlich in der ganzen Mundhöhle, namentlich auf der Zunge und im Rachen zu suchen. Als Folge derselben könnte man die abnormen, in der Mundhöhle sich geltend machenden Gährungen ansehen. Die saure Gährung würde von einem aus Kohlenhydraten, die faulige von einem aus Albuminaten bestehenden Nährmaterial der Pilze abzuleiten sein. Dass die specifischen Gährungserreger auch direct mit den Nahrungsmitteln in den Mund gelangen können, ist selbstverständlich. Wenn jedoch bei gleichmässiger, unveränderter, bisher gesunder Diät, wie bei Säuglingen an der Mutterbrust, plötzlich Indigestionserscheinungen mit saurer Reaction der Mundhöhle auftreten, findet man fast stets entzündliche Veränderungen am Zahnfleisch.

Die faulige Gährung der Mundhöhle nimmt, mit Ausnahme von Verletzungen oder specifischen Entzündungen der Mund- und Rachenhöhle, gewöhnlich ihren Anfang von entzündetem Zahnfleisch. Als nächster Ausdruck dieser Gährungen treten Veränderungen an der Zunge auf; bei der sauren ein grauer immer dicker werdender, bei der fauligen ein schmutzig gelblicher immer dünner werdender Belag, durch welchen stellenweise die rothe, zum Austrocknen geneigte und glänzende Schleimhaut durchblickt. Von der Zunge verbreitet sich der Process längs der Mandeln auf die Rachenhöhle. Die unausbleibliche Folge des Ergriffenseins jener mit Geschmacksnerven ausgestatteten Organe ist Störung des Appetits, welcher bei der sauren Gährung noch theilweise erhalten, aber auf scharfe, reizende Speisen gerichtet ist, bei der fauligen in Widerwillen gegen Nahrungsaufnahme ausartet, namentlich gegen Fleischspeisen, den man gewissermaassen für instinctiv halten könnte, um den Mikroben kein Nährmaterial zuzuführen.

Die noch ziemlich allgemein geltende Vorstellung, dass die Zunge. ein Spiegel des Magens, und dass im letzteren der Grund des Appetitmangels zu suchen sei, sollte wohl endlich dem Grundsat?. Platz machen, dass für die Frage des Appetits lediglich der Zustand der Zunge und Rachenhöhle entscheidend ist.

Bei reiner Zunge sieht man auch in fieberhaften Krankheiten den Appetit erhalten, z. B. bei Phthisikern, welche ihres Hustens halber fleissig die Mund- und Rachenhöhle mit Getränken durchspülen. Wie andererseits bei darniederliegendem Appetit die Magenverdauung sehr wohl erhalten sein kann, beweisen die günstigen Erfolge der Sondenfütterung selbst solcher Kranken, welche beim blossen Versuch, Fleischspeisen zu schlingen, von Brechwürgen befallen werden. Die Vomiturition wird demnach nicht vom Magen, sondern vom kranken Rachen her ausgelöst, und lässt sich das Widerstreben der natürlichen Nahrungsaufnahne beseitigen, wenn man auf fleissige Desinfection der Mundund Rachenhöhle hält. Die Weigerung der Aufnahme selbst flüssiger Nahrung bei schweren fiebernden, z. B. typhösen Kranken liegt sicherlich viel weniger in der Benommenheit ihres Sensoriums, denn sie trinken ja recht begierig eingeflösstes Wasser, als in ihrer durch

85. Bd. III. Abtheil. Aprilheft 1882. - Ueber die Richtung des Einathmungsstromes in der Nasenhöhle. Mitheilungen für den Verein Schleswig-Holsteinischer Aerzte 1885. 10. Heft. Stück 7. 15. März. 
Verunreinigung der Mund- und Rachenhöhle bedingten totalen Appetitlosigkeit. Desinficirt man diese Organe recht fleissig durch Darreichung eines nicht widerlich schmeckenden Mittels; wozu sich am besten die vorerwähnte einprocentige Kochsalzlösung mit einem halben Procent Jodtinctur (alle halbe Stunde wenigstens einen lalben Esslöffel) eignet, so gelangt man bald dazu, solchen Kranken einige Liter Buttermilcl oder verdünnte Milch den Tag über beizubringen.

Seit 5 Jahren wende ich dieses Mittel lediglich zur Reinlaltung der oberen Verdauungswege bei allen fiebernden Kranken an, olne dass ich jemals von den zuweilen Wochen lang fortgesetzten Gaben voll gegen zwei Gramm Jodtinctur auf 24 Stunden einen Nachtheil bemerkt hätte. Zunge und Rachen halten sich bei dieser Behandlung rein und feucht, die fuliginösen Beläge schwinden und erscheinen höchstens gegen Morgen, wenn das Mittel während des Schlafes längere Zeit ausgesetzt worden. Die Jod-Chlornatriumlösung hat einen entschiedenen Vorzug vor der sonst in solchen Fällen beliebten Salzsäurelösung, welche nicht uur weniger energisch desinficirend wirkt, sondern auch für die Dauer nicht ertragen wird und, was erst später zu Tage tritt, die Kranken um ihre Zähne bringt.

In fieberlosen Krankheiten kanı ansser der erwähnten Lösung der Jodtinctur in Kochsalzlösung eine solche in 10 Theilen Baldrian-, Myrrhen-, Guajac-, Eucalyptus-, Zimmet-, Galläpfeltinctur zur Desinficirung der Mund- und Rachenhöhle benutzt werden, wovon man jedesmal etwa 20 Tropfen zu einem Gläschen Wasser thut. Da auch der innere Gebrauch dieser hier seltener darzureichenden Mittel (2-3-6 stündlich) unschädlich ist, so wird den Kranken empfohlen, nur den ersten Schluck des Desinfectionsmittels nach erfolgter Spülung der Mund- und Rachenhöhle auszuspeien, den Rest langsam gurgelnd $\mathrm{zu}$ verschlucken, um die tieferen Rachentheile zu erreichen und auch den Magen gleichzeitig zu desinficiren. Des Morgens ist es rathsam, vor der ersten Spülung die Zähne und das Zahnfleisch mit einer mit der gewöhnlichen Toilettenseife bestrichenen Gummibürste (die Borstenbürsten verletzen leicht das Zahnfleisch) von anhaftendem Schleim zu reinigen. Künstliche Gebisse müssen vor jeder Spülung herausgenommen und abgespült, im Verlaufe fieberhafter Krankheit unbedingt für die ganze Dauer derselben entfernt werden.

Cariöse Zähne müssen, wenn sie nicht gut plombirt werden können, herausgezogen werden, weil sie fortwährend das Zahnfleisch und die Mundhöhle inficiren, und die Reinhaltung der Mundhöhle im höchsten Grade erschweren, wenn nicht gar illusorisch machen.

Dass die in der Mundhöhle wuchernden Mikroben nebst ihren Producten in den Magen gelangen, ist ein unausbleiblicher Vorgang. Es lässt sich wohl annehmen, dass ein normal functionirender Magen die meisten Eindringlinge mit verdauen wird, ebenso jedoch, dass wenn es ihm entweder unter der Last übermässiger schwer verdaulicher Speisen oder dem Hemmschuh eines chronischen Siechthums an der erforderlichen Muskel-Energie oder an genügendem Magensaft zur Verarbeitung seines Inhalts mangeln wird, die fremdartigen Fermente äber das normale die Oberhand gewinnen und den ganzen Verdauungsprocess stören werden, was sich durch Druck, schmerzhafte Contractionen des Magens, Auftreibung des Epigastriums, Aufstossen von Gasen und saurer Flüssigkeit, Uebelkeit, zuweilen selbst Erbrechen mit den bekannten reflectorischen Kopfschmerzen, allgemeiner Nervenaufregung und Schlaflosigkeit dem Eigenthümer fühlbar macht. Diese dyspeptischen Beschwerden werden gewöhnlich auf Rechnung eines Magenkatarrhs geschrieben; oder man hat auch die Existenz einer nervösen Dyspepsie zugelassen.

So nahe es liegt, perverser Innervation einen hervorragenden Einfluss auf die Magen-Verdauung zuzuerkennen, um nur an die Digestionsstörungen zu erinnern, welche nach heftigen, so körperlichen wie psychischen Eindrücken, während der Menstrual- und Schwangerschaftsperioden, im Verlauf von Erkrankungen der Sexualorgane, namentlich aber auf anämischem Boden aufzutreten pflegen, und welche man auf Anomalien der Verdauungssäfte oder abnorme Bewegungen des Verdauungskanals zurückzuführen sucht, so glaube ich doch Anhalt dafür gefunden zu haben, dass auch für diese Fälle von Dyspepsie sich grösstentheils eine aus der Mundhöhle stammende Infection des Magens herleiten liesse. Sehr häufig mache ich die Beobachtung, dass an hochgradiger Chlorose leidende junge Mädchen mit vollkommen gesunden Zähnen und unversehrtem Zahnfleisch, keinerlei Störungen der Magenverdauung unterliegen, abgesehen von dem Hang nach Säuren und absorbirenden Mitteln; während solche mit kranker Mundhöhle nicht nur allen möglichen Verdauungsbeschwerden ausgesetzt sind, sondern auch gewöhnlich die sonst so hülfreichen Eisenmittel schlecht vertragen.

Wenn man sich daran erinnert, dass anämische Personen, namentlich Frauen, während der Geschlechtsphasen so häufig an Zahnschmerzen leiden, dass in diese Zeit hauptsächlich der Beginn der cariösen Processe fällt, so dürfte man in der hier vorwaltenden sauren Reaction der Mundhöhle ebenso wie beim Gebrauch von flüssigen Eisenmitteln die Vorbedingung finden, unter welcher sich Mikroparasiten im Zahnfleisch und den Zähnen einnisten, und von hier aus den Mageninhalt inficiren können.

Bekanntlich werden in Fällen von mit Digestionsbeschwerden complicirter Chloranämie mehr die Salzsäure, bittere Mittel, Pepsin empfohlen und unstreitig günstigere Erfolge als mit reinen Eisenmitteln gewonnen. Indessen habe ich gefunden, dass man viel schneller zum Ziele gelangt, wenn man vor jeder Mahlzeit die oberen Verdauungswege mit Jıd-Baldriantinctur desinficirt, und nach dem Essen ein Eisenpräparat in Pillenform nehmen lässt, welches dann gut vertragen wird. Was den als Hauptursache der Dyspepsie angeschuldigten Magenkatarrh anbetrifft, so mag derselbe in protrahirten Fällen recht häufig vorkommen, besonders bei Säufern. Wenn man aber erwägt, dass so grobe anatomische Läsionen des Magens, wie das runde Geschwür, der Krebs, welche doch gewöhnlich mit katarrhalischen Veränderungen der Magenschleimhaut vergesellschaftet gefunden werden, in denjenigen Krankheitsfällen, wo durch den Sitz der Affection die Wegsamkeit des Organs nicht beeinträchtigt wird, oft ganz occult ohne erhebliche Verdauungsstörungen verlaufen können; wenn man sich den überaus günstigen Erfolg vergegenwärtigt, welchen Ausspülungen des Magens in den exquisitesten Magenkatarrhen z. B. bei der Ectasie schaffen, so dürfte man doch dazı gedrängt werden, die Ursache der Verdauungsbeschwerden weniger in der Magenschleimhaut, als vielmehr in dem Inhalt des Magens zu suchen. Auch die günstige Wirkung der anderweitigen, innern Arzneimittel gegen die aus vermeintlichem Magenkatarrh hergeleitete Dyspepsie, wie z. B. der angeblich schleimlösenden Alkalien, der bitteren Mittel, ja selbst des Salzherings, dieses Solamen potatorum, würde ganz ungezwungen die Erklärung zulassen, dass alle diese Mittel, die Alkalien durch chemische Umstimmung des Nährbodens, die Amara, der Salzhering mit seinem Gehalt an Kochsalz und Trimethylamin doch wohl mehr gegen die Gährungserreger des Mageninhalts als auf die Magenschleimhaut einzuwirken geeignet sind. Den Salzhering, ein an und für sich gewiss keineswegs leicht verdauliches Nahrungsmittel, habe ich nicht nur von Trinkern, sondern auch von anderen im höchsten Grade erschöpften Kranken z. B. im Endstadium des Morbus Brightii, deren rebellischer Magen keinen Löffel Suppe bei sich behielt, Wochen lang als einziges, instinctiv verlangtes Nahrungsmittel gut vertragen sehen, und kann ich mir diesen Zusammenhang nicht anders erklären, als dass er recht dazu angethan ist, während des Kauens und Schlingens die ganzen oberen Verdauungswege und den Magen selbst gründlich zu desinficiren. Unter Berücksichtigung all dieser Umstände glanbe ich annehmen zu können, dass jegliche Verdaunngsstörungen auf abnormen Gährungsvorgängen des Mageninhalts beruhen, die allerdings wohl durch Blutstasen der Magenwand und abnorme Innervation begünstigt werden können, dass der Magenkatarrh an sich aber mehr eine Folge als Ursache derselben ist.

Dass die pathologischen Gährungserreger ausser aus anderen Quellen auch aus Entzündungsheerden der Mund- und Rachenhöhle, namentlich aber des bisher für so harmlos gehaltenen Zahnfleisches entstammen können ${ }^{1}$ ), dafür könnte ich eine zahllose Casuistik anführen, will hier aber nur einige seit Jahren unter meinen Augen verbleibende Fälle kurz skizziren.

1. Eine Dame von 50 Jahren, welche bis auf eine lang bestehende Aorten-Insufficienz gesund, sich stets der kräftigsten Verdauung zu erfreuen hatte, fing nach einem schweren Gram unter Nachlass des Appetits, über nach jeder Nahrungsaufnahme eintretende Cardialgien, Aufstossen, Sodbrennen an zu klagen, so dass sie nur leichte flüssige Speisen, zuletzt nur Thee ohne Beschwerden vertragen konnte. Mehrmals wiederholte Kuren mit Karlsbader Brunnen brachten nur kurz anhaltende Besserung. Ein übler Geruch aus dem Munde, der sich namentlich in den Frühstunden bemerkbar machte, liess mich endlich in der Mundhöhle, die übrigens mit gesunden oder ausgezeichnet plombirten Zähnen besetzt war, eine Schwellung und leichte Ulceration des Zahnfleisches entdecken. Ausserdem war die Zunge stets belegt und die hintere Rachenwand etwas geröthet.

Fleissige Spülungen der Mund - und Rachenhöhle mit JodMyrrhen-Tinctur beseitigten biunen einigen Tagen die Cardialgien und brachten den früheren Appetit wieder.

Sobald die im höchsten Grade arzneischeue Dame wenn auch nur einen Tag die Desinfection der Mundhöhle unterlässt, schwillt das stets etwas hyperämische Zahnfleisch von Neuem, und die frühe-

1) Bisher ist mir nur eine von Ewald in seinem Artikel über Eiweissverluste beiläufig gemachte Bemerkung bekannt, dass die Kur mancher Magenkrankheit, so trivial das klänge, mit der Aufrăumung kranker Zähne zu beginnen habe. Nachträgliche Bemerkung des Autors.

$34[\mathrm{a}]$ 
ren Verdauungsbeschwerden melden sich wieder. Dieses Spiel von Magenbeschwerden im Gefolge recrudescirender Zahnfleisch-Entzündung habe ich Gelegenheit schon 7 Jahre lang zu beobachten.

2. Ein 40 jähriger Gymnasiallehrer, welcher schon seit seiner Studentenzeit an schlechter Verdauung, wie schmerzhaftem Druck in der Magengegend nach dem Essen, heftigen Kopfschmerzen, Schlaflosigkeit gelitten und zur vorsichtigsten Diät verdammt war, verfiel vor 4 Jahren in einen Zustand totaler Appetitlosigkeit, heftiger Magenschmerzen selbst nach flüssiger Nahrung, die nervösen Begleiterscheinungen äusserster Dyspepsie warfen ihn aufs Lager, auf welchem er nach 2 Monaten im höchsten Grade erschöpft, sein sicheres Ende nahe glaubte. Damals zu ihm berufen, wurde ich sofort höchst unangenehm durch einen widerlich sauren Geruch aus seinem Munde berührt, und falld das sämmtliche Zahnfleisch längs der ziemlich gut erhaltenen Zähne im höchsten Grade geröthet, aufgequollen, leicht blutend, an den Rändern schwärend. Patient gestand, schon seit seinen Jugendjahren leicht aus dem Zahnfleisch geblutet, aber keine Schmerzen daran empfunden zu haben, und urgirte auch diesmal nur seine Magenbeschwerden, hochgradiges Schwächegefühl und Schlaflosigkeit. Schon nach 24 Stunden, während welcher alle 15 Minuten die Jod-Kochsalzlösung genommen wurde, minderte sich der Ekel gegen alle Speisen, in 8 Tagen liessen unter Gebrauch der vorher unerträglichen Milch sämmtliche Verdauungsbeschwerden nach, nach 14 Tagen, nachdem das sehr gefässreiche Zahnfleisch mit Jod-Galläpfeltinctur vollständig ausgeheilt worden, wurden bei lebhaftestem $\Lambda$ ppetit auch alle anderen Speisen gut vertragen, und erfreut sich seitdem der früher in der Auswahl der Speisen sehr ängstliche Herr der untadelhaftesten Verdauung, aber nur so lange, als er regelmässig vor jeder Mahlzeit seine Mundhöhle mit Jod-Myrrhentinctur desinficirt. Jede Unterbrechung dieser Vorsicht, wie auf Reisen, oder aus Nachlässigkeit rächt sich sofort durch Wiederauftauchen der Zahnfleisch-Entzündung, welcher dyspeptische Beschwerden auf den Fusse folgen, was während 4 Jahren dreimal vorgekommen ist. Bei dieser Gelegenheit hätte ich noch zu erwähnen, dass ich den bei älteren Personen so häufig vorkommenden Widerwillen gegen süsse Milch, so wie die durch dieselbe hervorgerufenen Verdaungsbeschwerden (Pyrose, Diarrhoe, Appetitverlust) fast ausschliesslich in entzündlichen Zuständen der Mund- und Rachenhöhle, besonders aber des Zahnfleisches, welche die Milch mit Gährungserregern schwängern, begründet glaube, indem unter regelmässiger vorangeliender Desinfection dieser Höhlen die Bedenken des sonst erwünschten Milchgenusses bald umgangen werden können.

3) Eine 70 Jahre alte, seit einem Jahre wegen Inanition und grösster Schwäche bettlägerige, bis zum Skelett abgemagerte Frau hatte während dieser Zeit wegen Widerwillen gegen andere Speisen und Schmerzen im Magen noch denselben, ausschliesslich von Kaffee gehabt. In beiden Kiefern steckten nach vorne sechs wackelnde Zähne, umgeben von stark geschwollenem, ulcerirendem Zahnfleisch. Die Zunge war roth und trocken, ebenso der Rachen. Die Herzgrube auf Druck sehr empfindlich, der Unterleib aufgetrieben, der Stuhl angelalten. Die Frau hielt sich bei ihrer grossen Schwäche für gelähmt an den Beinen. Nach Entfernung der Zähne und Ausheilung des Zahnfleisches mit Jod - Myrrhentinctur schwand die Entzündung der Mund- und Rachenhöhle, es stellte sich sehr lebhafter $\Lambda$ ppetit ein, die allmählich dreister gegriffene Kost wurde sehr gut vertragen, so dass die alte Frau nach sechs Wochen geheilt und ziemlich fest auf den Beinen die Anstalt verlassen konnte.

Bald nach den Weihnachtsfeiertagen, während welcher süsse, klebrige Sachen, wie Pfefferkuchen und Marzipan, welche an den Zähnen haften bleiben, im Uebermaass genossen werden, begegne ich stets einer grossen Auzahl acuter Gastricismen, welche mit acuter Gingivitis einhergehen und aller Wahrscheinlichkeit durch dieselbe hervorgerufen werden.

Chronische Dyspepsien nehmen bei vielen vorher kerngesunden Menschen ihren Anfang von dem Zeitpnnkt, nachdem sie künstliche Gebisse, welche nicht genügend reingehalten werden, angelegt liaben. $\Lambda$ Is Belag hierfür will ich von hunderten Fällen nur den folgenden, keinen Zweifel über den Zusammenhang zulassenden anführen.

4) Ein 50 Jahre alter, musterhaft gebauter nud ernährter Gutsbesitzer, welcher niemals in seinem Leben krank gewesen sein wollte, hatte seit einigen Jahren nach jeder Mittagsmahlzeit über eine lästige $\Lambda$ ufgetriebenheit der Magengegeud zu klagen, welche erst nach einigen Stunden unter reichlichem $\Lambda$ ufstossen von Gasen vorüberzugeher pflegte. Die Untersuchung ergab ein künstliches Gebiss des Oberkiefers, welches der Inhaber seit zwei Jahren gar nicht gerührt hatte nach Entfernung desselben eine starke Röthung und $\Lambda$ uflockerung des Zahnfleisches und harten Gaumens, sowie eine Vergrösserung des linken, auf Druck leicht empfindlichen Leberlappens. Nachdem sich der Patient gewöhnt hatte, das Gebiss 1lach jeder Mahlzeit zu entfernen, und die Mundhöhle gründlich zu desinficiren, schwanden ohne weiteres Zuthun die Digestionsbeschwerden, und nach vier Wochen, als ich ihn wiedersah, war auch die Leberschwellung nicht mehr zu constatiren.

Nicht selten begegnet man im Gefolge von Zahnfleischentzündungen, namentlich bei Personen, welche künstliche Gebisse tragen und die Mundtoilette vernachlässigen, hartnäckigen Kehlkopfsentzündungen, asthmatischen Beschwerden, welche jeder Behandlung trotzen, bevor nicht in der Mundhöhle gründlich aufgeräumt wird.

5) Im Jahre 1875 hatte ich bereits über ein junges Mädchen berichtet, welches seit neun Jahren über einen äusserst quälenden Husten, Heiserkeit, sehr reichlichen schleimig-eitrigen, zuweilen etwas blutig gefärbten Auswurf klagte, in Folge dessen abzumagern anfing und von melireren $\Lambda$ erzten für schwindsüchtig erklärt worden war. Bei der näheren Untersuchung stellte sich herans, dass die Dame ein künstliches Gebiss trug, welches sie seit einem Jahre niemals aus der Mundhöhle entfernt hatte. Nach $\Lambda$ bhebung desselben präsentirte sich eine ausgedehnte Ulceration des ganzen oberen Kieferrandes, die Schleimhaut des harten Gaumens war tiefroth, mit grauen Einsprenkelungen und wie ein Schwamm aufgequollen, die ganze Rachenwand bis zum Kehlkopfseingang glänzend geröthet, die Stimmbänder schmutzig roth verfärbt und am Rande des rechten eine graue, -stecknadelkopfgrosse Ulceration mit Substanzverlust. Die penibelste Localtherapie der afficirten Schleimhäute hatte niclit eher Erfolg, bis die auf ihre Zähne eitle Dame nach vergeblichen Heilversuchen schliesslich meiner Mahnung Folge leistete, das künstliche Gebiss auf vierzehn Tage ganz bei Seite zu legen, worauf dann unter fleissiger Desinfection der Mundhölle und Inhalationen mit Jod-Galläpfeltinctur sämmtliche Beschwerden bis auf eine etwas rauhe Stimme, die Folge eines geringen Defectes anı rechtel Stimmbande, nachliessen und später auch nach $\Lambda$ nlegung des Gebisses bei nunmehr scrupulöser Reiuhaltung der Mundhöhle nicht wiederkehrten.

Die grauen Einsprenkelungen des Gaumens ergaben sich als Einlagerungen vol1 Oidium albicans, welches aller Wahrscheinlichkeit nach auch die Ulceration des Stimmbandes hervorgerufen hatte.

6) Eine 42 Jahre alte Dame litt seit zwei Jahren an äusserst heftigen Hustenparoxysmen, welche namentlich in der Rückenlage auftraten, den Schlaf raubten und zuweilen eine bis zu Ohnmachtsanfällen sicl steigernde Erschöpfung zur Folge hatten. Diese von vieIen Aerztell, wegen Complication mit metritisclien Erscheinungen, für hysterisch gehaltenen Beschwerden trotzten den verschiedenen Curversuchen und wurden nur durch Cliloralhydrat einigermaassen in Schranken gehalten. Objectiv war an der lintern Rachenwand eine leichte streifige Röthung, die sich bis auf den Kehlkopfseingang fortsetzte, nacl $\Lambda$ blegung eines gerade seit zwei Jahren getragenen künstlichell Gebisses, welches Patientin auf Anrathen des Zahnarztes höchst selten aus dem Munde entfernt hatte, starke Röthung des oberen Zahnfleisches und des ganzen Gaumens wahrzunehmen, von welcher sich Ausläufer bis nach dem Rachen verfolgen liesselı. Nach $\Lambda$ usheilung der Mund- und Rachenhöhle, zu welchem Zweck die küustlichen Zälne 8 Tage lang bei Seite gelegt wurden, liessen die Hustenparoxysmen nach und sind seitdem niclit wiedergekehrt.

Was die Propagation voll Muudkrankheiten auf das Olır durch die Tuba Eustachii betrifft, so will ich mich nur auf den Ausspruch des amerikanischen Ohrenarztes Saxton bernfen, welcher auf $1000 \mathrm{Er}$ krankungen des innern Ohrs $600 \mathrm{mal}$ die Ursache in kranken Zähnen gefunden, was im Grunde hauptsächlich auf Entzündungen des Zahnfleisches zurückzuführen ist.

Um den Zusammenhnng von Zahufleischentzündungen und $\Lambda \mathrm{u}-$ genkrankheiten zu illustriren, will ich mich nur auf einen mich selbst betreffenden Fall beschränken, der nicht wenig dazu beigetragen, meine ganze Aufmerksamkeit der ätiologischell Bedeutung von Zahnund Zahnfleischkranklieiten zuzuwenden. Im Jahre $1877^{\circ}$ acquirirte ich aus unbekannter Ursache eine parenchymatöse Keratitis des linken $\Lambda$ uges, welche vom äussern Rande in verticaler Richtung langsam fortschritt und nach der Ansicht mehrerer oculistischer Celebritäten wohl unaufhaltsain über die ganze Horlhaut sich ausbreiten sollte. Als bereits zwei Drittel der Hornhaut durch Exsudat getrübt waren, und gegen $\Lambda$ bend die wüthendsten Ciliar-, Supra- und Infraorbitalneuralgien Wochen lang wiederkehrten, merkte ich endlich, dass dem Ausbruch der Schmerzen ein Brenmen im Zahnfleisch beider oberen vorderen linksseitigen Backenzähne voraufging, von denen der zweite wegen Caries vor einem Jahre plombirt war mit Hinterlassung eines Bohrlochs zur Ventilation der Pulpa. Instinctiv einen Zusammenhang zwischen dem Zalın und meinen Neuralgiell fühlend, liess ich den plombirten Zahn herausziehen, worauf ich nach vier Wochen die erste, schmerzfreie Nacht hatte. Nach zwei Tagen 
kehrte jedoch der Infraorbitalschmerz wieder, auch diesmal angekündigt durch ein Brennen im Zahnfleisch des ersten, im Uebrigen ganz intacten Backenzahnes. Nach Entfernung auch dieses, vollkommen gesunden, aber von stark entzündetem Zahnfleisch wallartig umgebenen Zahnes schwanden nicht nur alle Schmerzen, sondern auch der Entzündungsprocess der Hornhaut, gegen welchen die heroischsten Curen vergeblich angewendet wordell waren, fing von jenem Tage ab, unter allmählicher Wiederkehr der bis dahil erloschenen Sensibilität der Conjunctiva bulbi, seinen Rückzug anzutreten an, das $\mathbf{P a}$ renchym der Hornhaut klärte sich allmählich, und im späteren Verlauf kam es nur noch zu oberflächlichen, partiellen Infiltraten anf der stark vascularisirten Oberfläche der Hornhaut. Zur Aufklärung dieses eigenthümlichen Zusammenhanges habe ich anzufübren, dass die Pulpa des plombirten zweiten Backenzahnes kurz vor dem Ausbruch der Keratitis gangränös geworden, wie aus dem üblen Geruch der zur Wegsamhaltung des Bohrlochs eingeführten hölzernen Sonden zu schliessen war. Es ist nun möglich. dass die aus dem Zahne ausfliessende Jauche das Zahnfleisch in nächster Umgebung inficirt und längs der Nervenscheiden des entsprechenden Trigeminusästchens fortkriechend eine Neuritis ascendens desselben hervorgerufen, in Folge deren die die Conjunctiva bulbi versorgenden Fasern anästhetisch geworden und so der Infection der Cornea Vorschub geleistet hatten. Die Reizung der Nervenendigungen im entzündeten Zahnfleisch beider Zähne kann einerseits durch Reflex auf die Vasomotoren der Hornhaut das Fortschreiten der Keratitis begünstigt, andererseits die periodischen Irradiationen auf die Ciliar- und Orbitalnerven ausgelöst haben, so dass mit Aufhebung des Reizzustandes durch Extraction beider Zähne zuerst die Neuralgien im Augengebiet und darauf der entzündliche Process in der Cornea selbst sistirt wurde.

\section{(Schluss folgt.)}

V. Arnold. Ueber Kerntheilung nnd vielkernige Zellen. Virch. Arch. 98. S. 501.

Krauss. Beiträge zur Riesenzellenbildung in epithelialen Geweben. Ib. 95 . S. 249

Orthman. Ueber Tuberculose der weiblichen Brustdrüse mit besonderer Berücksichtigung der Riesenzellenbildung. Ib. 100 . S. 365 .

Naegeli. Ueber den Einfluss der Pilze auf die Bildung von Riesenzellen. Dissert. Bern 1884. Ref. Ribbert.

Neben der Kerntheilung durch indirecte Segmentirung hat Arnold in mehreren früheren Arbeiten auch eine solche durch indirecte Fragmentirung beschrieben, bei welcher der Kern sich in ein aus dicken Bändern zusammengesetztes Knänel umwandelt, das dann in mehrere Fragmente zerfällt. Aehnliche Beob̆achtungen hat Verf. nun auch bezüglich der Karyokinese in Riesenzellen gemacht. Er untersuchte zunächst die vielkernigen Zellen in Sarcomen und Carcinomen und fand hier beide Methoden der Theilung nebeneinander vertreten. In hyperplastischen Lymphdrüsen machte er an den vielkernigen Gebilden die gleichen Erfahrungen. Fernerhin sah er auch in den scrophulösen Drüsen Kernfiguren innerhalb der Riesenzellen, die darauf hindeuten, dass auch die Randstellung der Kerne sich auf Grund der indirecten Fragmentirung ausbilden kann. Arnold betont, dass diese Thatsache seine frühere Auffassung modificire, nach welcher die Tuberkelriesenzellen scrophulöser Drüsen vorwiegend durch Confluenz kleinerer Zellen entstanden gedacht werden mussten.

Ein derartiges Zusammenfliessen von Zellen kommt in Geschwülsten nach den Mittheilungen von Krauss für die Bildung von Riesenzellen sehr wesentlich in Betracht. $\mathrm{Er}$ untersuchte epitheliale Tumoren und fand zunächst in einer Reihe gewöhnlicher Epitheliome in directer Beziehung $\mathrm{zu}$ den epithelialen Zapfen grosse vielkernige Zellen, die zum Theil ganz im Innern derselbell lagen und offenbar durch Confluenz benachbarter Epithelien entstanden waren. Es ging das neben anderen Gründen aus der gleichen Beschaffenheit des Protoplasmas und auch daraus hervor, dass die Zellen nach aussen sich nicht immer scharf begrenzen liessen, sondern allmählich in das umgebende Protoplasma des Zapfens übergingen. Andere Riesenzellen grenzten an das Bindegewebe an, noch andere lagen anscheinend frei in demselben. Jedoch war diese Erscheinung zum Theil nur durch die Schnittricktung bedingt, womit aber die Entstehung aus dem Bindegewebe nicht überhaupt in Abrede gestellt werden soll. Reichliche Riesenzellen fand Verf. ferner um Ligaturfäden im Innern eines Krebses und sah auch die Entstehung derselben aus confluirenden Epithelien in einem Talgdrüsenadenom.

Ueber das Zusammenfliessen von Epithelzellen zu Tuberkelriesenzellen berichtet weiterhin $0 \mathrm{rthmann}$ in zwei Fällen von Tuberculose der weiblichen Brustdrüse, in denen die Auffindung von Bacillen gelang. Der eine von ihnen war als primäre Erkrankung der Mamma aufzufassen, da andere Organe nicht verändert waren, der zweite dagegen war complicirt mit tuberculöser Pericarditis, Verkäsung von Bronchialdrüsen etc., so dass der Krankheitsbeginn nur mit einiger Wahrscheinlichkeit in die Brustdrüse verlegt werden konnte. Riesenzellen wurden nun sowohl in dem die Drüsenräume umgebenden Granulationsgewebe, hier aus epithelioiden Zellen hervorgehend, gefunden, wie innerhalb der Acini und Ausführungsgänge. An letzteren Orten liess sich aus verschiedenen Umständen ein Zusammenfliessen der von der Wand sich ablösenden Epithelien zu Riesenzellen erschliessen. So waren die Kerne zuweilen in mehrere kreisförmige Figuren angeordnet, die einen Schluss auf die Vereinigung benachbarter Drüsenbläschen zu einer Riesenzelle gestatteten. In den Ausführungsgängen fanden sich oft lang hingezogene Formell, die den Kanal auf längere Strecken ganz ausfüllten.

Die Bildung von Riesenzellen bei den tuberculösen Processen wird bekanntlich auf die Einwirkung von Bacillen zurückgeführt, die im Inneren derselben sich finden und auch von Orthmann hier nachgewiesen wurden. $\mathrm{Ob}$ auch andersartige Riesenzellen unter dem Einfluss von Spaltpilzen entstehen, hat Naegeli zu entscheiden gesucht. Er benutzte eine frühere Beobachtung von Langhans (Virch. Arch. 49), nach welcher in der Umgebung von Blutextravasaten bei Tauben sich Riesenzellen entwickeln, die offenbar zur Resorption des Blutes in Beziehung stehen. Verf. machte nun zwei Reihen von Versuchen. In der einen wurde das Extravasat absichtlich mit faulenden Substanzen inficirt, in der anderen vollkommen aseptisch verfahren. Auf die Resultate im Einzelnen, die Umwandlung des Blutkuchens etc. geht Ref. hier nicht ein, als wichtigstes Resultat ist dagegen zu verzeichnen, dass Riesenzellen nur bei Gegenwart von Spaltpilzen, nicht in den aseptischen Versuchen beobachtet wurden.

Die Mikroben wurden aber nicht wie bei der Tuberculose in den Riesenzellen, auch nicht in dem umgebenden entzündeten Gewebe, wo sie offenbar rasch zu Grunde gehen, gefunden, sondern nur in dem Blutkuchen, der also wohl einen günstigen Nährboden bildet. Sie kommen demnach für die Riesenzellenbildung hauptsächlich durch ihre chemische Einwirkung in Betracht. Trotz der Prägnanz seiner Resultate möchte Verf. nun noch nicht schliessen, dass Spaltpilze zur Bildung von Riesenzellen stets nothwendig seien, aber eine grosse Bedeutung kommt ihnen zweifellos zu.

VI. Jahresbericht über die Fortschritte der Pharmacotherapie. Ein Nachschlagebuch für Lehrer der medicinischen Wissellschaften, Aerzte und Apotheker: Von Dr. Kobert, Assistenten des pharmacologischen Institutes der Universität Strassburg. I. Band für das Jahr 1884. Strassburg, Verlag von Karl J. Trübner. 1885. Ref. Buchwald.

Wir begrüssen dieses Jahrbuch des geschätzten Verfassers, welches die wichtigsten Lehrbücher, Monographien und Aufsätze aus dem Gebiete der Therapie in gedrängter Uebersicht aufzählt, resp. kritisch bespricht, mit Freuden. Bei dem grossen Umfange des Materials hat Kobert vorläufig seine Aufmerksamkeit besonders auf die Nervenund Muskelgifte, welche gegenwärtig das Feld beherrschen, gerichtet, manche andere Gruppe noch unberücksichtigt lassend. Von der Anführung beliebter Receptformeln hat er ganz Abstand genommen, ein Vorgang, den wir nur billigen können. Solche Formeln sind nur geeignet, das eigene Denken und Forschen zu verhindern. Bei der Eintheilung wählt er die wissenschaftliche Sonderung in physiologische Gruppen nach dem Vorbilde von Schmiedeberg, Buchheim, Harnack. Nachdem zuerst die Lehrbücher über allgemeine und specielle Therapie aufgezählt sind, werden von den Nerven- und Muskelgiften die Gruppen des Strychnins, Codeins, Curarins, Morphins, Alcohols etc. ausführlich besprochen. Der erste Theil schliesst mit Cannabinin. Der Practiker wird sich allerdings erst gewöhnen müssen, manche Präparate aus bekannten Mitteln in verschiedenen Gruppen aufzusuchen, so Tetano-Cannabin, Cannabinin, Cannabinon, Cannabin etc. in verschiedenen Abtheilungen, doch gewinnt er durch diese kleine Mühe gleich das Verständniss für die physiologische Wirksamkeit. Das Buch kann jedem Arzte; der sich wissenschaftlich mit der Pharmacotherapie beschäftigt, auf das Angelegentlichste empfohlen werden. Die Ausstattung ist eine vorzügliche.

VII. Die Arzneimittel der heutigen Medicin für die ärztliche Praxis, zusammengestellt von Dr. med. Otto Roth. 5. Auflage, von Dr. Gregor Schmitt neu bearbeitet. Würzburg. A. Stuber's Verlagshandlung. 1885. 342 Seiten. Klein Octav. Ref. Buchwald.

Das Buch, welches innerhalb 10 Jahren 5 Auflagen erlebt, ent- 
spricht gewiss einem Bedürfniss der practischen Aerzte. Es bietet auch dem Arzte in gedrängter Kürze so viel des Wissenswerthen, dass sein Erfolg auch für die 5. Auflage gesichert sein wird. Nach einer kurzen Einreihung der Arzneimittel in Gruppen, werden die einzelnen Stoffe alphabetisch aufgeführt. Es sind dabei die meisten älteren und neueren Mittel, wie Cocain, Cannabin, Hydıarg. formamidatum, Kairin, Thallin etc. erwähnt und ihre Anwendungsweise zweckentsprechend hervorgehoben. Bei vielen Mitteln sind gangbare, oder von hervorragenden Aerzten bevorzugte Formeln eingereiht, die subcutane und Inhalationstlerapie wird genügend berücksichtigt. Winke für eine billige Verschreibweise in der Armenpraxis werden gegeben. In dem umfangreichen 8. Capitel "Therapentische Notizen ${ }^{\text {" }}$ wird die Behandlungsweise der einzelnen Krankheiten, im 9. Capitel die Dermatotherapie, im 10. die Behandlung von Vergiftungen besprochen. Das 11. Capitel, wo einige Daten über Herzdämpfung, Percussion der Milz etc. gegeben werden, ist sehr kurz; es könnte wohl ganz wegbleiben, da es mit der sonstigen Tendenz des Buches nichts zu thun hat. Die Ausstattung ist eine gute.

VIII. Ueber die habituelle Obstipation und ihre Behandlung mit Electricität, Massage und Wasser von Dr. Georg Hünerfauth. (Besitzer der Wasserheil-Anstalt zu Bad Homburg.) Wiesbaden, Verlag von J. F. Bergmann. 1885. Oct. 50 Seiten. Ref. Buchwald.

Das kleine Werk giebt in den ersten beiden Capiteln die bekannten Ursachen und Symptome der habituellen Verstopfung in grossen Zügen an. Wo die bekannten Laxantien und Adstringentien ihre Dienste versagen, feiert die combinirte electrisch-hydropathische und Massagebehandlung noch grosse Triumple. Die 3 Methoden werden entweder einzeln oder combinirt in Anwendung gezogen: sie wirken ja alle 3 auf Anregung der Nerven und Muskelthätigkeit des Darmes, stärkeren Zufluss von Verdauungssäften, Beseitigung von Circulationsstörungen, sowie Kräftigung der Bauchpresse. Bè der electrischen Behandlung wird der faradische, der constante Strom und die Galvanofaradisation nach de Watteville (Mendel's Neurol. Centralbl. 1882. No. 12) angewendet. Die Galvanofaradisation is besonders wirksam und verdient von den Aerzten melır berücksichtigt zu werden. Je nach der Natur des Leidens wechselt man mit der Grösse der Electroden und dem Orte ihrer Application (Rectum, Unterleib, Medulla).

Bei der Massage kommen Streichung, Knetung, Drückung, Klatschung, Erschiitterung in Betracht. Die Massage des Unterleibs will geübt sein, ist aber dann ungefährlich und von ausgezeichneter Wirkung. In nicht sachkundiger Hand kann sie nachtheilig wirken.

Bezüglich der Wassercuren erwähnt Verfasser, dass Abreibung, Douche, Sitzbad, Klystiere und nasse Leibbinden den meisten Erfolg erkennen lassen. Die Applicationsmethode und die Temperatur des Wassers muss für jeden einzelnen Fall sorgfältig ausgeprobt werden. Man geht im Allgemeinen von wärmeren Graden zu kälteren über. Besonders gute Erfolge sieht man nach den auf den Unterleib gerichteten mobilen horizontalen Regendouchen und mobilen Fächerdouchen, sowie von der Anwendung kurzer (10 Minuten lang) kalter $\left(10^{\circ}\right.$ Réaumur) Sitzbäder.

Einzelne Krankengeschichten sind aufgeführt. Das kleine Buch bietet dem practischen Arzte einen recht gaten Anhalt für die Behandlung des hartnäckigen Leidens.

IX. P. Fürbringer. Die Krankheiten der Harn- und Geschlechtsorgane. Braulschweig, Wreden's Sammlung kurzer medicinischer Lehrbücher. VIII. 1884. Ref. Kopp-München.

In wirklich musterhafter Weise ist der Verfasser der ihm gestellten Aufgabe, ein kurzgefasstes und doch alles Wesentliche enthaltendes Lehrbuch der Harnkrankheiten zu schaffen, gerecht geworden, und es scheint mir damit eine längst von Studirenden und Aerzten empfundene Lïcke in der sonst so reichhaltigen medicinischen Literatur ausgefüllt. Mit ganz besonderem Talente und einer Klarheit der Darstellung, welche gerade in dieser Materie selten zu finden ist, hat F. das Capitel der Nierenkrankheiten bearbeitet, indem er mehr auf eine schnelle und präcise Orientirung in den hauptsächlichsten klinisch feststehenden Thatsachen, als auf die Darstellung derjenigen theoretis chen Anschauungen, welche bis heute noch nicht genïgend gefestigt er schienen, Gewicht gelegt hat; gleichwohl hat auch aus dem rein theoretischen Gebiete das Wichtigste ausreichende Beobachtung gefunden. Zweifellos von grossem Nutzen besonders fïr den Anfänger und Studenten erscheint mir der das Lehrbuch eröffnende allgemeine Theil, in welchem die Albuminurie, Hämaturie, Hämoglobinurie, die Harncylinder, Hydrops und Urämie nach Wesen, Formen und Ursachen abgehandelt werden; dadurch wird das Verständniss der folgenden
Capitel ausserordentlich erleichtert. In dem speciellen Theil des Buches werden dann zunächst die Erkrallkungen der Nieren besprochen und zwar die Kreislaufsstörungen (Choleraniere, Schwangerschaftsniere, Stauungsniere und der hämorrhagische Niereninfarct), dann die Nierenentzündungen, und die Geschwülste und die Lageveränderungen der Nieren. Die Besprechung der eigentlichen Nephritis, von welcher die reine Stauungsniere, sowie die Cholera- und Schwangerschaftsniere getrennt wird, beginnt mit einer kurzen aber klaren geschichtlichen Einleitung. Als Eintheilungsprincip werden die feineren histologischen Unterschiede bei den Nierenbefundell, welche nur verschiedenen Stadien einer Erkrankung entsprechen, obgleicl zugegeben wird, dass nicht iı jedem einzelnen Falle alle Stadien mit genügender Schärfe in Erscleinung treten, als unberechtigt zurückgewiesen, und $\mathrm{F} \ddot{\mathrm{r}} \mathrm{r}$ bringer nimmt als eine den klinischen Thatsachen am besten entsprechende Classification die drei wollcharakterisirten Krankheitsbilder der acuten und chronischen diffusen Nephritis und der vollendeten Schrumpfniere an. Die Darstellung dieser drei Hauptformen ist eine in jeder Hinsicht gründliche und klare. Aus den folgenden Abschnitten, Amyloidniere, Nephritis suppurativa, Pyelitis und Pyelonephritis, Peri- und Paranephritis, Nephrolithiasis und dem Capitel über die Nierengeschwülste haben wir nichts wesentlich Nenes hervorzuheben. Das Gleiche gilt von den Lageveränderungen des Organes. Dann werdell die Erkrankungell der Blase abgehandelt, und soll hier insbesondere auf das interessante Capitel der Enuresis nocturna verwiesen werden.

Als ein Lehrbuch der Geschlechtskrankheiten im Grossen und Ganzen aber kann man das Fürbringer'sche Buch kaum betrachten; denn nur der Tripper und seine Complicationen, und die Erkrankungen der samenbereitenden und Samen leitenden Gebilde, sowie die Neuralgieen der männlichen Genitalien haben hier eine in practischer Hinsicht gewiss sehr werthvolle Bearbeitung gefunden. Für den Tripper mag man ja mit Rücksicht auf die Häufigkeit complicatorischer Erscheinungen von Seiten des uropoetischen Systems die Aufuahme ill ein Lehrbuch der Harnkrankheiten wohl begreiflich finden; dasselbe gilt für die Stricturen. Epididymitis und Balanitis aber, Prostatitis, männliche Impotenz und Sterilität stehen, wie mir scheint, mit den Erkrankungen der Harnorgane in einem zu losen Zusammenhange, um mit denselben gemeinsam in einem Buche besprochen zu werden, zumal wenn die ganze Reilıe der ïbrigen Genitalaffectionen des Mannes und des Weibes, wie in diesem Falle, unberücksichtigt bleiben mussten. Man könnte daher den letzten Theil des Buches besser als eine Sammlung einiger ausgewählter Capitel der Lehre von den Genitalaffectionen bezeichnen, bei deren Auswahl der Autor durch eine gewisse rein specialistische Vorliebe geleitet wurde. Darum wollen wir das Buch nicht weniger allen denen empfehlen, welchen es um eine schnelle und präcise Orientirung auf dem hier behandelten Gebiete zu thun ist, und es wird dem heutzutage mit Arbeiten so sehr überhäuften jungen Mediciner die Lectüre desselben sicherlich von grossem Nutzen sein. 